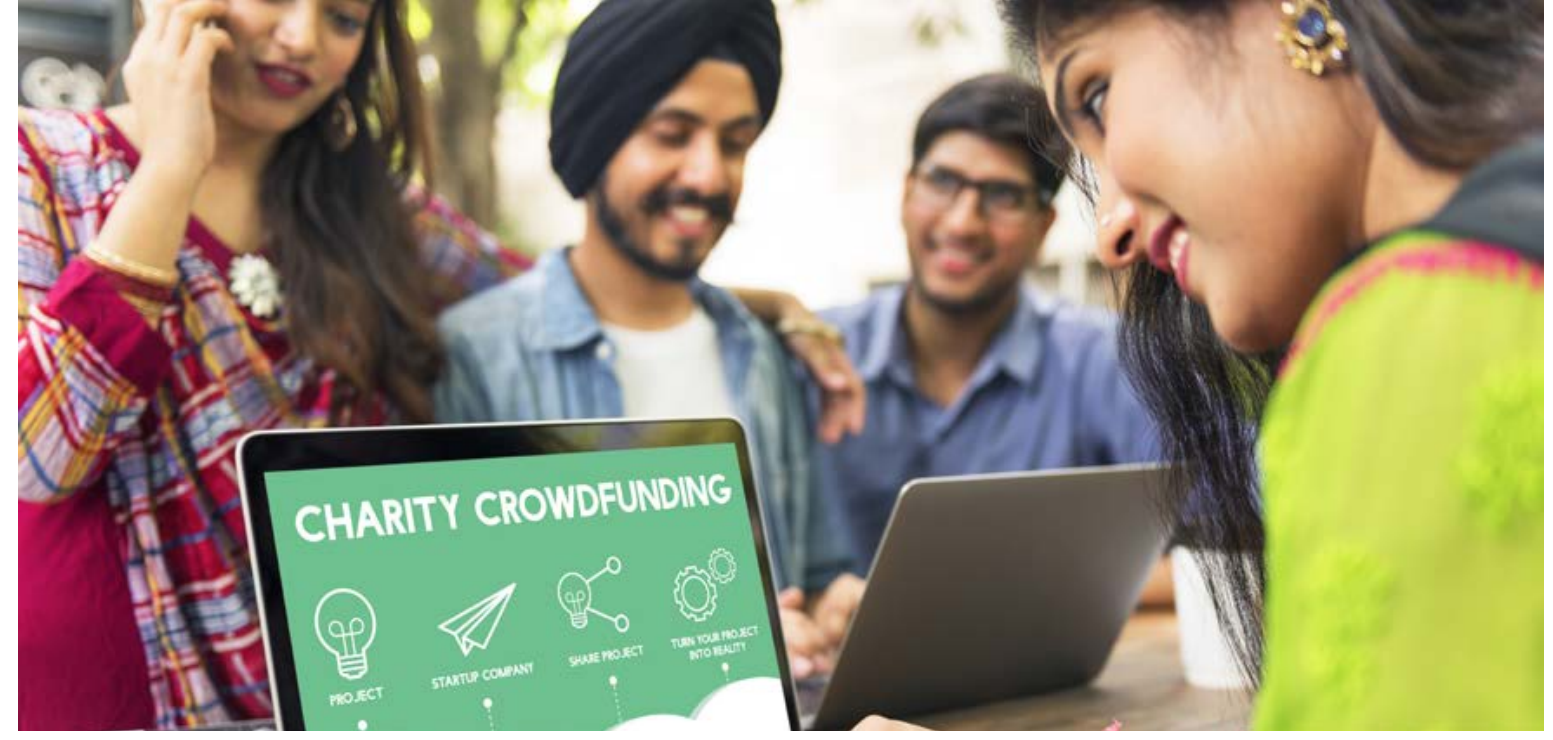

\title{
El emprendimiento y su impacto social positivo
}

DOl: http://dx.doi.org/10.18259/per.2019001

\author{
Raj K Shankar* \\ Division of Innovation and Entrepreneurship, Nord University Business School
}

\begin{abstract}
La creciente demanda de relevancia de los efectos sociales dentro del emprendimiento (Wiklund et al., 2019) ha provocado que las últimas investigaciones académicas se orienten a explorar los enfoques empresariales para responder a exigencias sociales, como son la acción empresarial frente a los desastres naturales, el alivio de la pobreza y la búsqueda del bienestar humano.
\end{abstract}

$\mathrm{E}$ I emprendimiento es ampliamente considerado como una fuerza poderosa para el desarrollo socioeconómico (Urbano, Aparicio \& Audretsch, 2019). Incluso, cuando las referencias más populares al espíritu empresarial hablan sobre la creación de empleo, la generación de riqueza y el desarrollo regional, el espíritu empresarial ha tenido gran impacto social positivo, a través del alivio de la pobreza (Sutter, Bruton \& Chen, 2019) y del sufrimiento (Shepherd \& Williams, 2018), mejora de la educación superior (Nabi, Liñán, Fayolle, Krueger \& Walmsley, 2017), avance de la sostenibilidad (Dean \& McMullen 2007), mejora del bienestar personal (Wiklund, Nikolaev, Shir, Foo y Bradley, 2019) y así como de la salud humana (Shepherd \& Patzelt, 2015).
Como lo destaca Shepherd (2015), la acción empresarial es una fuerza importante para abordar muchos de los grandes desafíos que enfrenta la sociedad actual. Por ello, la investigación sobre emprendimiento se ha expandido en el número de resultados o variables dependientes, más allá del desempeño de la empresa (Wiklund, Wright \& Zahra, 2019) y un vistazo rápido a los múltiples resultados del emprendimiento revela que, tratar en un solo artículo acerca de la influencia social positiva del emprendimiento, es casi imposible.

Por lo tanto, en esta oportunidad, haremos un modesto intento de aprovechar la vasta literatura académica sobre emprendimiento y revisaremos, brevemente, tres formas aprovechadas por la investigación sobre

Galardonado educador en emprendimiento. Obtuvo un posdoctorado en la División de Innovación y Emprendimiento, Nord University Business School, Noruega. Sus intereses de investigación abarcan los aceleradores corporativos, los ecosistemas empresariales y las oportunidades empresariales. 
emprendimiento para explorar los resultados sociales.

Esta aproximación es especialmente importante, sobre todo, debido a la creciente demanda de relevancia de los efectos sociales dentro del emprendimiento (Wiklund et al., 2019). El objetivo de este breve artículo, entonces, es señalar a los lectores artículos importantes y autores que sean pioneros en la investigación de estos aspectos importantes.

\section{Aventura espontánea y alivio del sufrimiento}

Dado que la frecuencia de los desastres naturales aumenta constantemente en todo el mundo, los efectos sociales negativos de tales desastres, así como los desafíos económicos, sociales y ambientales que imponen a su paso son cada vez más difíciles de comprender y abordar. Shepherd \& Williams (2018) extienden la literatura sobre la organización de la compasión al contexto del alivio de los desastres posteriores al sufrimiento.

Los mencionados autores definen la aventura espontánea, la organización local de acciones compasivas e improvisadas y la aparición rápida de una empresa compasiva como un enfoque empresarial para responder a desastres y sufrimientos.

Profundizan en dos tipos de desastres ocurridos en contextos diferentes: los incendios forestales ocurridos durante el año 2009 en Victoria (Australia) y el terremoto en Haití (en 2010), y presentan sus hallazgos a través de una serie de estudios (véase, por ejemplo, Williams \& Shepherd, 2016), a través de los cuales destacan cómo la aventura compasiva y espontánea es una forma única de emprender, ya que alivia el sufrimiento posterior al desastre. $Y$, ésta es solo una de las formas emergentes encontradas por los académicos del emprendimiento para explorar la relación entre el emprendimiento y el alivio del sufrimiento.

\section{El emprendimiento como solución a la pobreza}

Junto con el sufrimiento generado por los desastres naturales, la pobreza también es un desafío crítico que enfrentan la mayoría de las naciones en todo el mundo. Si bien se exploran soluciones disciplinarias para el alivio de la pobreza, en los últimos años, destaca el rol que, en este ámbito, ha ido asumiendo el espíritu empresarial.

La investigación sobre la vinculación de la pobreza y el espíritu empresarial es relativamente joven, pero está creciendo rápidamente. Bruton, Ketchen \& Irlanda ( 2013) y Wiklund et al. (2019) encuentran, potencialmente, tres formas de ver esta relación: remediación, reforma y revolución.

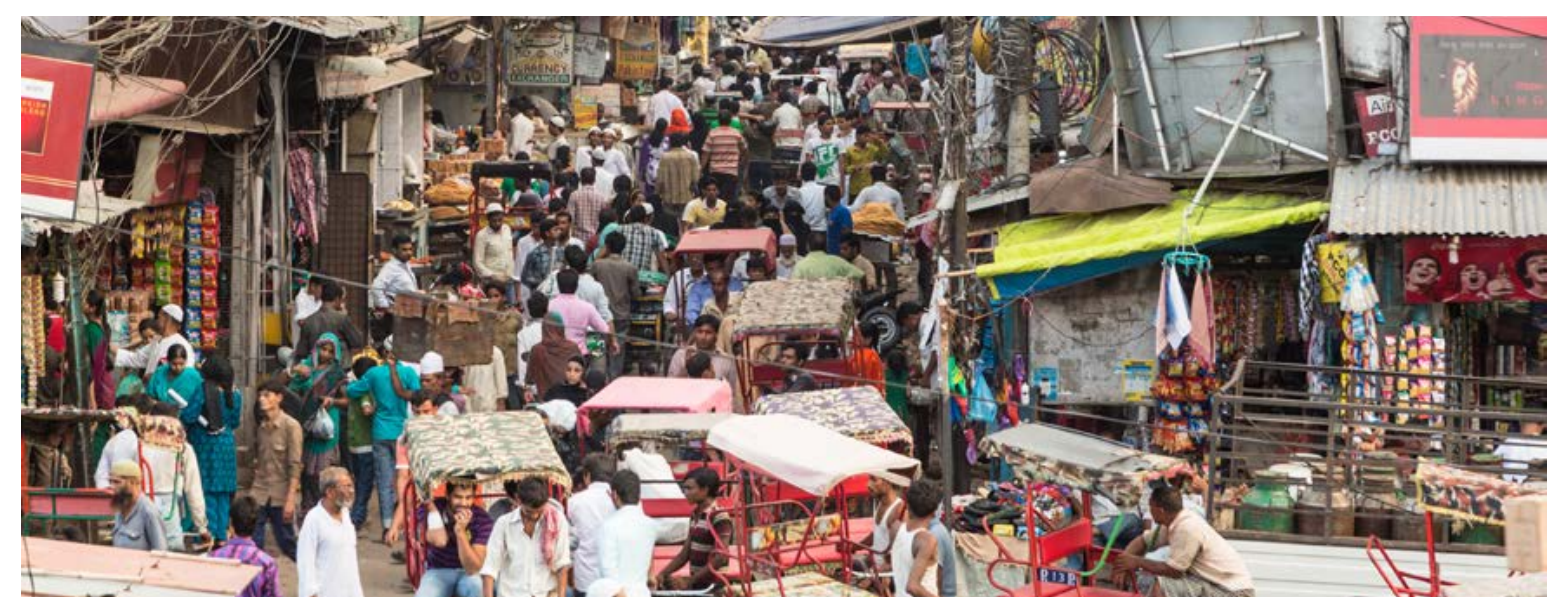


La perspectiva de "remediación» supone que la pobreza es el resultado de la escasez de recursos y que la provisión de recursos, por parte de las organizaciones de promoción y apoyo al emprendimiento, permitirán a los individuos y grupos participar en la formación de nuevas empresas, lo que eventualmente sacará a más personas de la pobreza.

Por su parte, la perspectiva de "reforma» supone que la pobreza surge debido a la exclusión social y, dado que el emprendimiento puede aumentar la inclusión, fomentar el emprendimiento y apoyar las iniciativas empresariales también puede aliviar la pobreza.

Finalmente, la perspectiva de la urevolución» supone que la pobreza es el resultado de sistemas corruptos y rotos, por lo que arreglar los a través de nuevas formas de organización social es una forma de reducir la pobreza. Si bien estas tres perspectivas no son la única forma de estudiar esta relación, resultan en una reducción de la pobreza al alentar la acción empresarial.

\section{Bienestar empresarial y un mundo más feliz}

A nivel mundial, la felicidad es actualmente aceptada como un objetivo humano fundamental. Y, desde el año 2013, las Naciones Unidas instauró el 20 de marzo como el Día Internacional de la Felicidad. Los individuos persiguen la vocación del emprendimiento por una variedad de razones idiosincráticas, y, dentro de este cúmulo de razones, las ganancias son un débil contendiente.

\section{C6 El espíritu empresarial es una fuerza poderosa para mejorar el bienestar general y el cociente de felicidad entre el fundador de la empresa y la sociedad."}

Experimentar la libertad, el control sobre su futuro, la realización personal y el desarrollo personal, es decir, todo lo relacionado con el bienestar, son razones que los empresarios suelen citar.

Y, si bien el espíritu empresarial puede mejorar el bienestar de los fundadores, también expande esa bonanza a sus familias y otras partes interesadas (empleados, proveedores, clientes, etc.). El emprendimiento y la nueva creación de valor resultante, entonces, pueden mejorar el bienestar de la sociedad en general.

Por consiguiente, parece que el espíritu empresarial es una fuerza poderosa para mejorar el bienestar general y el cociente de felicidad entre el fundador de la empresa y la sociedad. Las recientes convocatorias para avanzar en la investigación en la intersección del bienestar y el emprendimiento (Wiklund et al., 2019) destacan la necesidad de ver el bienestar como un resultado importante del emprendimiento, pues, explican cómo cambia el bienestar a lo largo del proceso emprendedor, caracterizado por su complejidad e incertidumbre, y exploran el

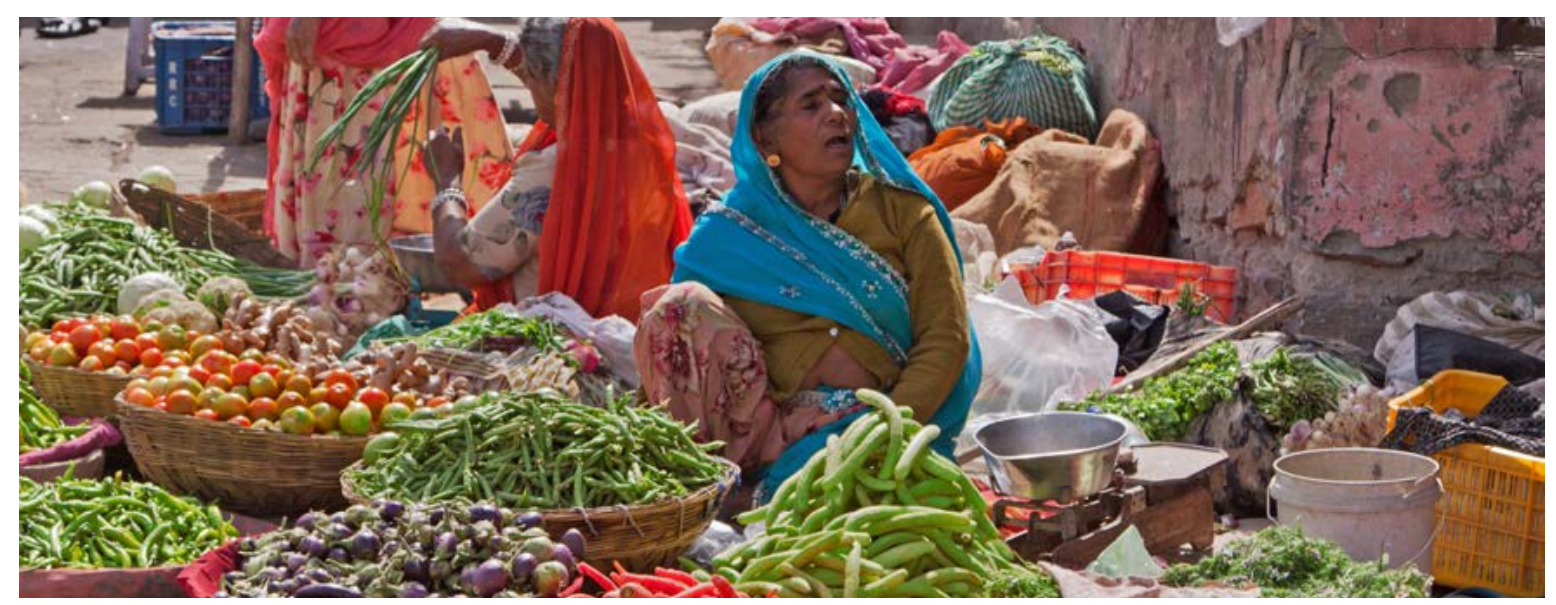


bienestar no sólo desde la óptica del emprendedor, sino de todos los involucrados en el proceso.

Incluso, destacan la importancia de estudiar el bienestar como un recurso psicológico, con influencias institucionales en el emprendimiento y el bienestar relacionado. Hay que precisar, además, que, tal como ocurre con la mayoría de los aspectos dentro del emprendimiento, estudiar el bienestar también es altamente contextual y requiere incluir conversaciones dentro de contextos socioeconómicos (Welter, 2011).

\section{Conclusión}

Como se explicó desde un inicio, el propósito de este documento es resaltar las numerosas formas en que el emprendimiento influye positivamente en la sociedad. Es así que, además de la creación de empleos y riqueza, hemos identificado al menos seis áreas en las cuales el emprendimiento ha tenido un tremendo impacto social positivo. Sin embargo, sólo hemos profundizado en tres: alivio del sufrimiento, alivio de la pobreza y bienestar. Esperamos, por tanto, que este breve artículo proporcione suficiente confianza sobre la influencia positiva del emprendimiento en la sociedad, así como una guía para sucesivas investigaciones en estas áreas, de modo que se prosiga con los avances obtenidos, tanto en la teoría como en la práctica del emprendimiento.

\section{Referencias}

Bruton, G. D., Ketchen Jr, D. J., \& Ireland, R. D. (2013). Entrepreneurship as a solution to poverty. Journal of Business Venturing, 28(6), 683-689.

Dean, T. J. \& McMullen, J. S. (2007). Toward a theory of sustainable entrepreneurship: Reducing environmental degradation through entrepreneurial action. Journal of Business Venturing, 22(1), 50-76.
Nabi, G., Liñán, F., Fayolle, A., Krueger, N. \& Walmsley, A. (2017). The impact of entrepreneurship education in higher education: A systematic review and research agenda. Academy of Management Learning \& Education, 16(2), 277-299.

Shepherd, D. A. (2015). Party On! A call for entrepreneurship research that is more interactive, activity based, cognitively hot, compassionate, and prosocial. Journal of Business Venturing, 30(4), 489-507.

Shepherd, D. A. \& Patzelt, H. (2015). The «heart» of entrepreneurship: The impact of entrepreneurial action on health and health on entrepreneurial action. Journal of Business Venturing Insights, 4, 22-29.

Shepherd, D. A. \& Williams, T., A. (2018). «Spontaneous Venturing: An Entrepreneurial Approach to Alleviating Suffering in the Aftermath of a Disaster». The MIT Press, Cambridge: Massachusetts.

Sutter, C., Bruton, G. D. \& Chen, J. (2019). Entrepreneurship as a solution to extreme poverty: A review and future research directions. Journal of Business Venturing, 34(1), 197-214.

Urbano, D., Aparicio, S. \& Audretsch, D. B. (2019). The Effect of Entrepreneurial Activity on Economic Growth. Institutions, Entrepreneurship, and Economic Performance (pp. 85-106). Springer, Cham.

Welter, F. (2011). Contextualizing entrepreneurship-conceptual challenges and ways forward. Entrepreneurship. Theory and Practice, 35(1), 165-184.

Wiklund, J., Wright, M., \& Zahra, S. A. (2019). Conquering relevance: Entrepreneurship research's grand challenge. Entrepreneurship Theory and Practice, 43(3) 419-436

Wiklund, J., Nikolaev, B., Shir, N., Foo, M. D. \& Bradley, S. (2019). Entrepreneurship and weIl-being: Past, present, and future. Journal of Business Venturing, 34(4), 579-588.

Williams, T. A. \& Shepherd, D. A. (2016). Building resilience or providing sustenance: Different paths of emergent ventures in the aftermath of the Haiti earthquake. Academy of Management Journal, 59, 2069-102. 